\title{
Tetrandrine suppresses $\beta$-glucan-induced macrophage activation via inhibiting NF-кB, ERK and STAT3 signaling pathways
}

\author{
$\mathrm{JING} \mathrm{XU}^{1}$, DABIAO LIU ${ }^{2}$, QING YIN ${ }^{1}$ and LANFANG GUO ${ }^{2}$ \\ ${ }^{1}$ Department of Laboratory Medicine, The Affiliated Hospital of Jiangsu University; ${ }^{2}$ Department of Laboratory Medicine, \\ The Affiliated Fourth People's Hospital of Jiangsu University, Zhenjiang, Jiangsu 212001, P.R. China
}

Received May 29, 2015; Accepted April 5, 2016

DOI: $10.3892 / \mathrm{mmr} .2016 .5187$

\begin{abstract}
Macrophages are important in inflammation through the production of various proinflammatory mediators. $\beta$-glucan is a polymer of glucose, which is produced by numerous different organisms, including fungi, and acts as a trigger for the induction of inflammatory responses. Tetrandrine (TET), a bis-benzylisoquinoline alkaloid isolated from the Chinese herb Radix Stephania tetrandra, has been demonstrated to modulate inflammatory responses. In the present study, it was investigated whether TET affects the inflammatory reaction induced by $\beta$-glucan in murine and human macrophages. It was demonstrated that $\beta$-glucan induced the activation of nuclear factor (NF)- $\kappa \mathrm{B}$ and markedly increased the levels of tumor necrosis factor- $\alpha(\mathrm{TNF}-\alpha)$ and interleukin $1 \beta$ (IL-1 $\beta$ ) in macrophages. Treatment with TET resulted in downregulation of phosphorylated NF- $\kappa \mathrm{B}$ p65 and reduction of the production of TNF- $\alpha$ and IL-1 $\beta$. In addition, the phosphorylation of ERK and STAT3 was decreased by TET in activated macrophages. Furthermore, it was demonstrated that the inhibitory effects of TET on $\beta$-glucan-induced macrophage activation was not due to its cytotoxic action. Conclusively, these results indicate that TET can decrease the inflammatory responses mediated by $\beta$-glucan in macrophages. Thus, TET may serve as an effective tool for the treatment of $\beta$-glucan-associated inflammatory diseases.
\end{abstract}

\section{Introduction}

Tetrandrine (TET) is a bis-benzylisoquinoline alkaloid extracted from the dried roots of Han-Fang-Chi (Stephania tetrandra S Moore). TET has a variety of biological activities and is traditionally used to treat patients with

Correspondence to: Ms. Lanfang Guo, Department of Laboratory Medicine, The Affiliated Fourth People's Hospital of Jiangsu University, 20 Zhengdong Road, Zhenjiang, Jiangsu 212001, P.R. China

E-mail: guolanfang415@163.com

Key words: tetrandrine, $\beta$-glucan, macrophage, nuclear factor- $\kappa \mathrm{B}$, inflammation fungal infection, silicosis or hypertension. The antitumor properties of TET have also been demonstrated $(1,2)$. Previous studies have suggested that TET reduces the inflammatory response by inhibiting the production of inflammatory mediators. In vitro studies have shown that TET inhibits cellular proliferation and cytokine production in activated monocytes and $T$ cells (3-5). TET suppresses nuclear factor (NF)- $\kappa B$ signaling pathways and reduces the production of tumor necrosis factor- $\alpha(\mathrm{TNF} \alpha)$, interleukin (IL)-1 $\beta$, IL-6 and NO in lipopolysaccharide (LPS) and amyloid $\beta$ (A $\beta)$-activated microglia (3-5). TET could also affect the mitogen-activated protein kinases signaling pathways, including extracellular signal-regulated kinase (ERK) in activated microglial and mast cells $(5,6)$. Therefore, TET has been suggested to be a potent anti-inflammatory agent. Macrophages are critical in the inflammatory response. Upon stimulation, macrophages display an activated state and produce proinflammatory mediators, such as TNF $\alpha$ and IL-1 $\beta$. Macrophage activation has been demonstrated in numerous inflammatory diseases. Therefore, targeting the proinflammatory activation of macrophages may have therapeutic potential.

$\beta$-glucans are polymers of glucose linked by $\beta$-glycosidic bonds. $\beta$-glucans are found in fungal cell walls, bacteria and plants. In recent years, a number of studies have shown that $\beta$-glucans can activate the immune system (7-9). $\beta$-glucans are recognized by their receptors, dectin-1 and toll-like receptor-2 in various cells $(10,11)$. Activation of the downstream pathways results in the induction of genes that activate the immune system. $\beta$-glucans have been shown to potently activate macrophages through activation of $\mathrm{NF}-\kappa \mathrm{B}$ (12). For those patients with infective diseases, $\beta$-glucans from fungal cell walls and bacteria may evoke macrophage activation and trigger the inflammatory response. Inhibition of $\beta$-glucan-mediated macrophage activation may represent a novel approach for treating inflammatory diseases. However, few studies have determined the effects of TET on $\beta$-glucan-induced macrophage activation.

In this study, the functional role of TET in $\beta$-glucan-activated macrophages and its possible mechanisms were investigated. It was demonstrated that pretreatment with TET inhibited IL-1 $\beta$ and TNF- $\alpha$ production by macrophages. These effects of TET may be attributed to its inhibitory effect on the NF- $\kappa \mathrm{B}$, ERK, signal transducer and activator of transcription 3 (STAT3) pathways during macrophage activation. The present 
Table I. Sequences of specific primers for reverse transcription-quantitative polymerase chain reaction.

\begin{tabular}{lllr}
\hline mRNA & Primer & \multicolumn{1}{c}{ Sequences $\left(5^{\prime}-3^{\prime}\right)$} & Tm $\left({ }^{\circ} \mathrm{C}\right)$ \\
\hline$\beta$-actin & Forward & CACGAAACTACCTTCAACTCC & 61 \\
& Reverse & CATACTCCTGCTTGCTGATC & 61 \\
TNF $\alpha$ & Forward & CCGAGTGACAAGCCTGTAGC & 61 \\
& Reverse & AGGAGGTTGACCTTGGTCTG & 61 \\
IL-1 $\beta$ & Forward & TACGAATCTCCGACCACCA & \\
& Reverse & GGACCAGACATCACCAAGC & \\
\hline
\end{tabular}

TNF, tumor necrosis factor; IL, interleukin.

findings highlight the clinical value of TET for the treatment of $\beta$-glucan-associated inflammatory diseases.

\section{Materials and methods}

Materials. $\beta$-glucan was purchased from Sigma-Aldrich (St. Louis, MO, USA). TET was purchased from PuZhen Biology (Shanghai, China). TET was dissolved in $0.1 \mathrm{M} \mathrm{HCl}$ and adjusted to $\mathrm{pH}$ 7.3.

Cell culture. Macrophage-like cells (murine RAW264.7 and human THP-1) were purchased from Cell Bank, Type Culture Collection Committee, Chinese Academy of Sciences (Shanghai, China). RAW264.7 macrophages were grown in high glucose Dulbecco's modified Eagle's medium (Thermo Fisher Scientific, Inc., Waltham, MA, USA) containing 10\% heat-inactivated fetal bovine serum (Thermo Fisher Scientific, Inc.). THP-1 cells were cultured in RPMI-1640 medium supplemented with $10 \% \mathrm{FBS}$. All the cells were grown at $37^{\circ} \mathrm{C}$ under a humidified atmosphere of $5 \% \mathrm{CO}_{2}$.

Cell viability assay. Cell viability was determined using an MTT (3-(4,5-dimethylthiazol-2-yl)-2,5-diphenyltetrazolium bromide) assay (Thermo Fisher Scientific, Inc.). Briefly, cells (3,000 RAW264.7 cells and 5,000 THP-1 cells) were seeded into 96-well plates overnight. TET was added at the indicted doses for $24 \mathrm{~h}(0,0.2,0.5 \mu \mathrm{M}, 1,2,5$ and $10 \mu \mathrm{M})$. Then, $10 \mu \mathrm{l}$ MTT solution $(5 \mathrm{mg} / \mathrm{ml})$ was added to each well and incubated for an additional $4 \mathrm{~h}$. Absorbance was measured at $570 \mathrm{~nm}$ using a microplate reader (Biotek, Winooski, VT, USA). Optical density was identified as the relative numbers of viable cells. All experiments were performed in triplicate.

Cell apoptosis assay. Cell apoptosis was measured by fluorescence-activated cell sorting (FACS) analyses (FACS Calibur; BD Biosciences, Franklin Lakes, NJ, USA) using the Annexin V-fluorescein isothiocyanate (FITC) Apoptosis kit (Thermo Fisher Scientific, Inc.) according to the manufacturer's protocol. Briefly, cells were seeded into 6-well plates overnight, then treated with various concentrations of TET $(0.2,0.5,1,2,5$ and $10 \mu \mathrm{M})$ for $24 \mathrm{~h}$. Cells were collected and resuspended in Annexin $\mathrm{V}$ binding buffer, stained with Annexin V-FITC and propidium iodide (PI), and analyzed by FACS. All samples were examined in triplicate.
Reverse transcription-quantitative polymerase chain reaction (RT-qPCR). Total RNA was isolated from cells using TRIzol Reagent (Invitrogen, Thermo Fisher Scientific, Inc.), according to the manufacturer's instructions. Equal quantities (1 $\mu \mathrm{g})$ of RNA was converted into cDAN using HiScript First Strand cDNA Synthesis kit (Vazyme, Nanjing, China). RT-qPCR was performed using SYBR Green I Real-time Detection kit (Cwbio, Beijing, China) on a Bio-Rad CFX96 Detection system (Bio-Rad Laboratories, Inc., Hercules, CA, USA). The relative expression was calculated using the $2^{-\Delta \Delta C q}$ method. $\beta$-actin was used as an internal control. The sequences of specific primers are listed in Table I. PCR conditions were as follows: $95^{\circ} \mathrm{C}$ for $5 \mathrm{~min}$, followed by 40 cycles of $94^{\circ} \mathrm{C}$ for $20 \mathrm{sec}$ and $61^{\circ} \mathrm{C}$ for $20 \mathrm{sec}$. All samples were examined in triplicate.

Western blotting. Cells were homogenized and lysed in radioimmunoprecipitation assay (RIPA) buffer supplemented with $1 \mathrm{mM}$ phenylmethanesulfonyl fluoride (PMSF, Sigma-Aldrich). Equal amounts of proteins were loaded and separated on a $10 \%$ sodium dodecyl sulfate-polyacrylamide gel electrophoresis gel (Thermo Fisher Scientific, Inc.) for electrophoresis followed by electro-transfer to polyvinylidene difluoride membranes (Thermo Fisher Scientific, Inc.). The membranes were blocked in 5\% (w/v) non-fat milk for $1 \mathrm{~h}$ and then incubated with primary antibodies overnight. The antibodies were obtained from the following sources: Antibodies against rabbit phosphorylated (p-)ERK (cat. no. 4376; 1:1,000), rabbit ERK (cat. no. 9102; 1:1,000), rabbit p-STAT3 (cat. no. 9145; 1:1,000), rabbit STAT3 (cat. no. 4904; 1:1,000), rabbit p-p65 (cat. no. 3033; 1:1,000) and rabbit p65 (cat. no. 4764; 1:1,000) were obtained from Cell Signaling Technology Inc. (Danvers, MA, USA); mouse anti-glyceraldehyde 3-phosphate dehydrogenase (GAPDH; cat. no. 1A6; 1;1,000), horseradish peroxidase (HRP)-conjugated goat anti-rabbit (cat. no. BS13278; 1:1,000) and goat anti-mouse (cat. no. BS50350) antibodies were purchased from Bioworld Technology (St. Louis Park, MN, USA). After washing with Tris-buffered saline (Thermo Fisher Scientific, Inc.) with Tween 3 times, the membrane was incubated with HRP conjugated secondary antibody and developed with enhanced chemiluminescence substrate (Thermo Fisher Scientific, Inc.) and imaged using an chemiluminescent system (LAS4000mini; GE Healthcare Life Sciences, Logan, UT, USA). The relative integrated intensity 
<smiles>COc1cc2c3cc1Oc1cc(ccc1C[C@H]1c4cc(cc(OC)c4OC)CCN1C)CCN(C)[C@H]3Cc1ccc(cc1)O2</smiles>

B

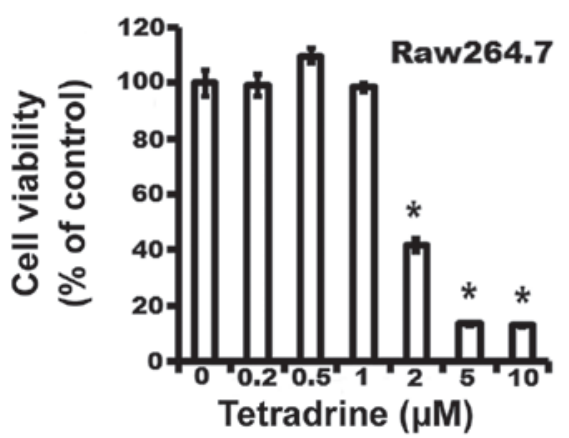

C

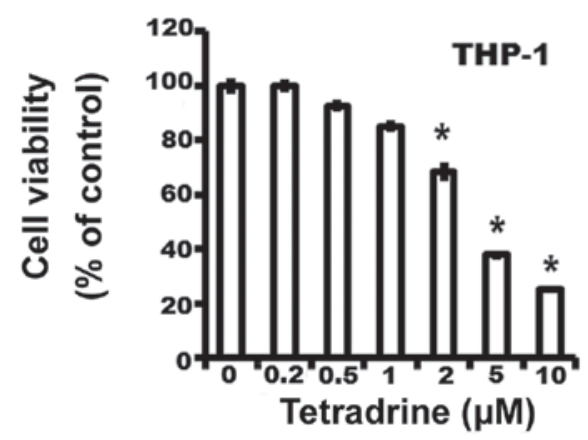

Figure 1. Effect of TET on the viability of Raw264.7 macrophages and THP-1 cells. (A) The molecular structure of TET. (B) Raw264.7 macrophages and (C) THP-1 cells were treated with various concentrations of TET $(0,0.2,0.5,1,2,5$ and $10 \mu \mathrm{M})$ for $24 \mathrm{~h}$. Cell viability was determined by an MTT assay. ${ }^{*} \mathrm{P}<0.05$, compared with $0 \mu \mathrm{M}$. TET, tetrandrine.

A
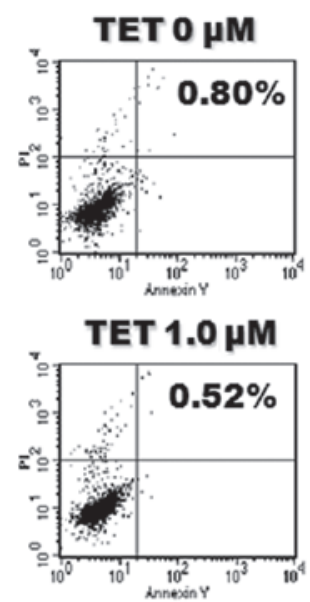

B

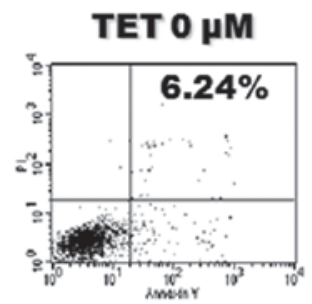

TET $1.0 \mu \mathrm{M}$

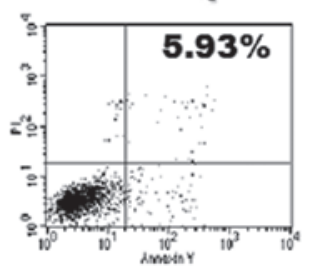

TET $0.2 \mu \mathrm{M}$

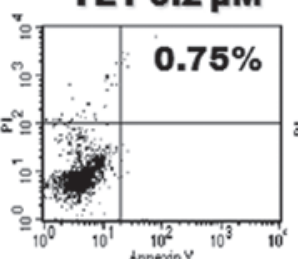

TET $0.2 \mu \mathrm{M}$

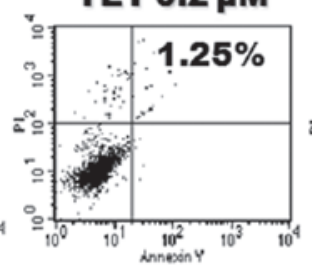

TET $0.2 \mu \mathrm{M}$

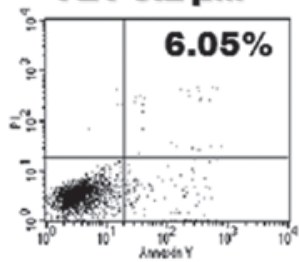

TET $0.2 \mu \mathrm{M}$

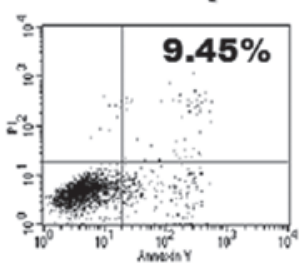

TET $0.5 \mu \mathrm{M}$

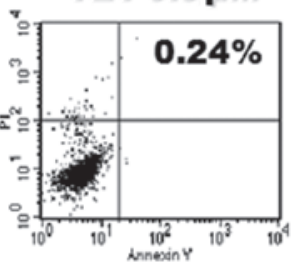

TET $0.5 \mu \mathrm{M}$

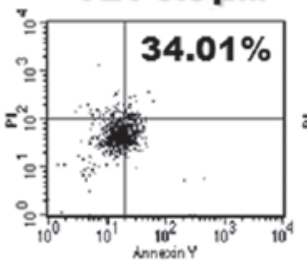

TET $10 \mu \mathrm{M}$

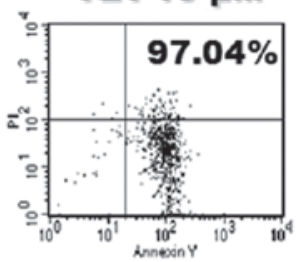

TET $0.5 \mu \mathrm{M}$

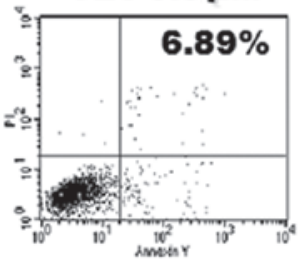

TET $0.5 \mu \mathrm{M}$

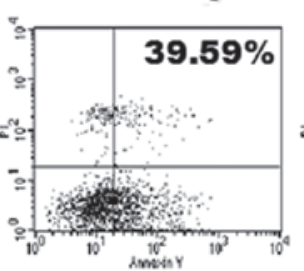

Raw264.7

THP-1

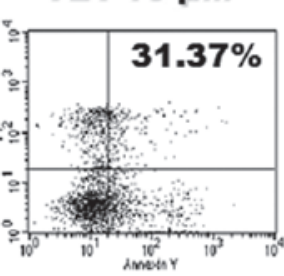

Figure 2. Effect of TET on the apoptosis of Raw264.7 macrophages and THP-1 cells. (A) Raw264.7 macrophages and (B) THP-1 cells were treated with various concentrations of TET $(0,0.2,0.5,1,2,5$ and $10 \mu \mathrm{M})$ for $24 \mathrm{~h}$. Cell apoptosis was detected by Annexin V/PI double staining followed by fluorescence-activated cell sorting analyses. TET, tetrandrine; PI, propidium iodide. 
A

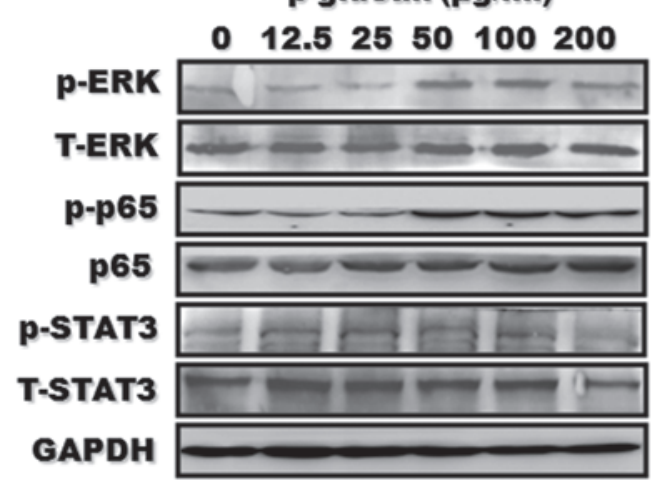

C

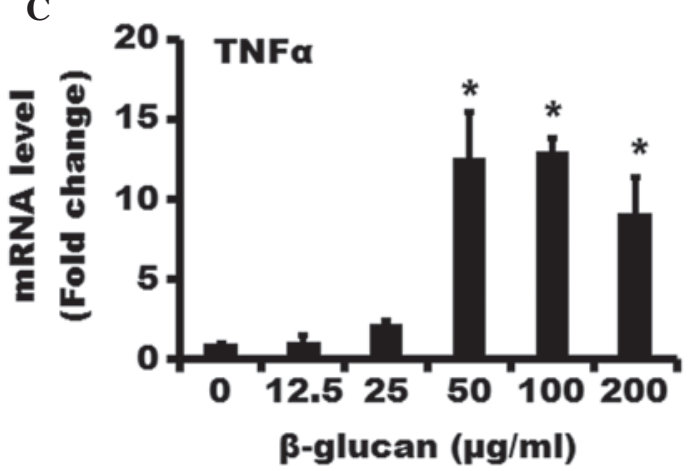

$\mathbf{E}$

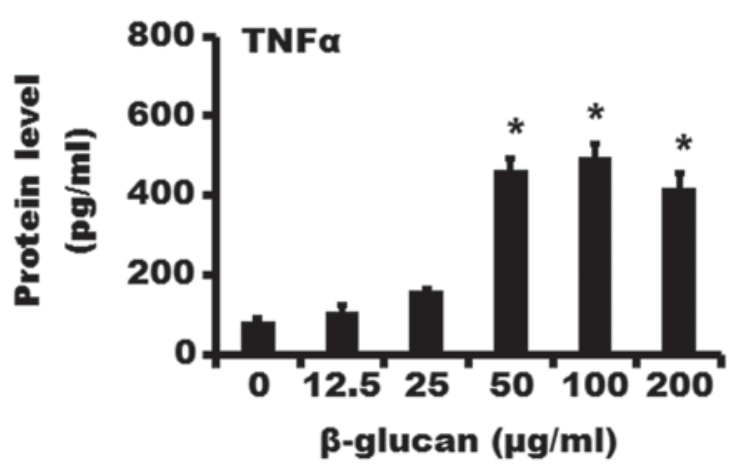

B

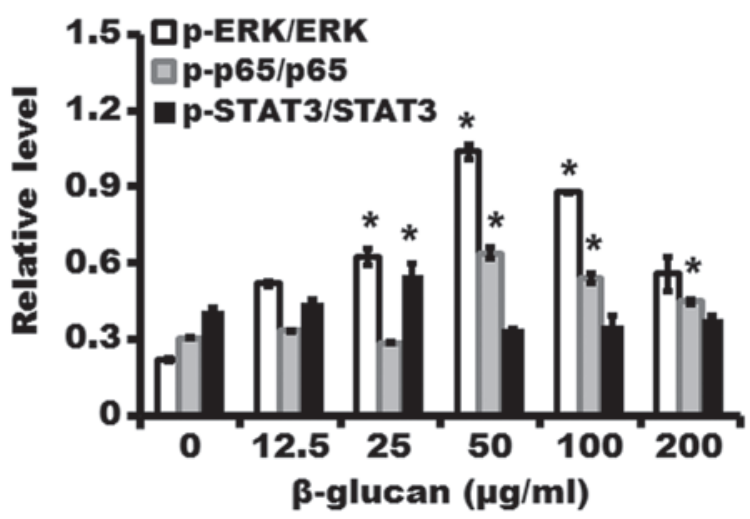

D

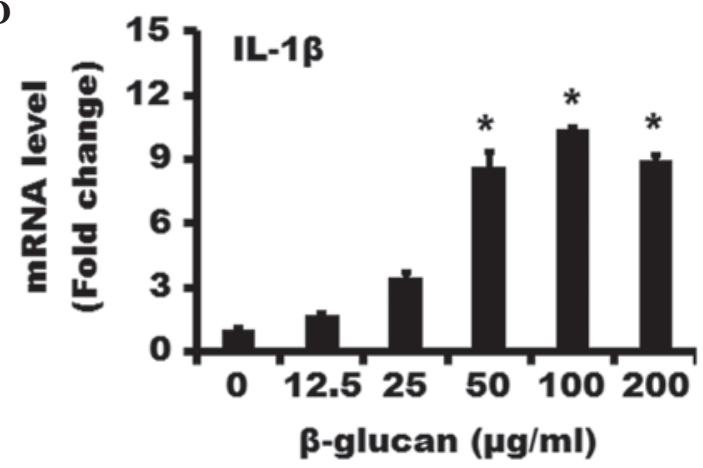

$\mathbf{F}$
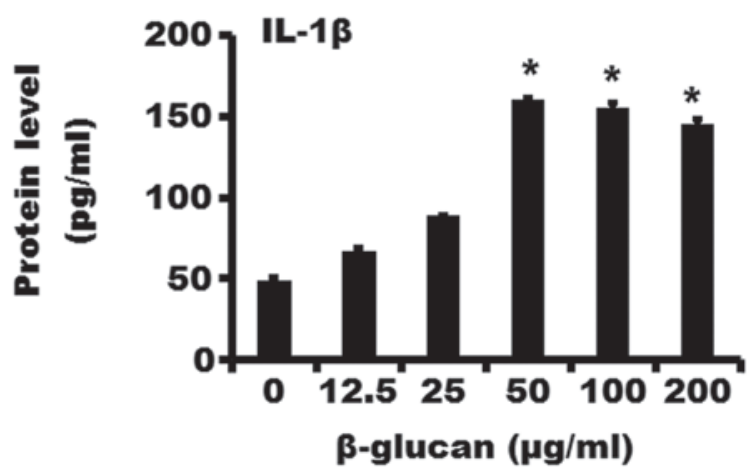

Figure 3. Effect of $\beta$-glucan on NF- $\kappa$ B, ERK and STAT3 pathways and expression of IL-1 $\beta$ and TNF $\alpha$ in THP-1 cells. THP-1 cells were treated with various concentrations of $\beta$-glucan $(0,12.5,25,50,100$ and $200 \mu \mathrm{g} / \mathrm{ml})$ for $24 \mathrm{~h}$. (A) The expression of phosphorylated and total forms of p65, ERK and STAT3 proteins were examined using western blotting. (B) The relative expression of p-p65/p65, p-ERK/ERK and p-STAT3/STAT3 was shown. The mRNA expression of (C) TNF $\alpha$ and (D) IL-1 $\beta$ in THP-1 cells treated with $\beta$-glucan at various concentrations for 24 h was examined by using revere transcription-quantitative polymerase chain reaction. The protein levels of (E) TNF $\alpha$ and (F) IL-1 $\beta$ in THP-1 cells treated with $\beta$-glucan at various concentrations for 24 h were examined by an enzyme-linked immunosorbent assay. ${ }^{*} \mathrm{P}<0.05$, compared with control. ERK, extracellular signal-regulated kinase; NF- $\kappa \mathrm{B}$, nuclear factor- $\kappa \mathrm{B}$; STAT3, signal transducer and activator of transcription 3; TNF $\alpha$, tumor necrosis factor- $\alpha$; IL, interleukin; p-, phosphorylated; GAPDH, glyceraldehyde 3-phosphate dehydrogenase; T-, total.

for p-p65, p65, p-ERK, ERK, p-STAT3, STAT3 was normalized to that of GAPDH in the same sample. Quantification was performed using ImageJ (version 2.0; National Institutes of Health, Bethesda, MD, USA). All tests were conducted in triplicate.

Immunofluorescence. Cells seeded on slides were pre-treated with various concentrations of TET for $2 \mathrm{~h}$, followed by incubation with $\beta$-glucan $(100 \mu \mathrm{g} / \mathrm{ml})$ for $24 \mathrm{~h}$. Cells were washed with PBS twice and fixed with $4 \%$ paraformaldehyde for $30 \mathrm{~min}$, permeabilized with $0.1 \%$ Triton X-100 for $10 \mathrm{~min}$, blocked with $5 \%$ bovine serum albumin (BSA), and then incubated with indicated primary antibodies at $4^{\circ} \mathrm{C}$ overnight followed by a $\mathrm{Cy} 3$-conjugated anti-rabbit secondary antibody. The cells were then counterstained with DAPI for $5 \mathrm{~min}$, and the images were acquired with a Nikon eclipse Ti-S microscope (Nikon, Tokyo, Japan).

Enzyme-linked immunosorbent assay (ELISA). The protein levels of TNF- $\alpha$ and IL-1 $\beta$ were measured using ELISA kits (Bangyi Biotech, Shanghai, China), according to the manufacturer's protocol. A volume of $100 \mu \mathrm{l}$ culture supernatants were added to each well and the absorbance was measured at $450 \mathrm{~nm}$. ELISA was performed in triplicate. 
A

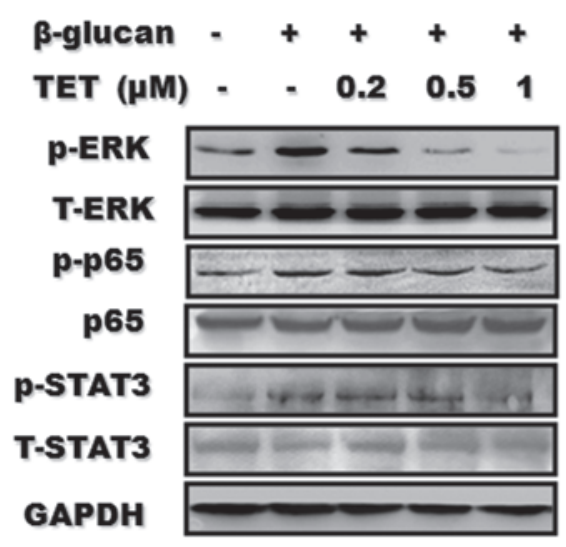

C

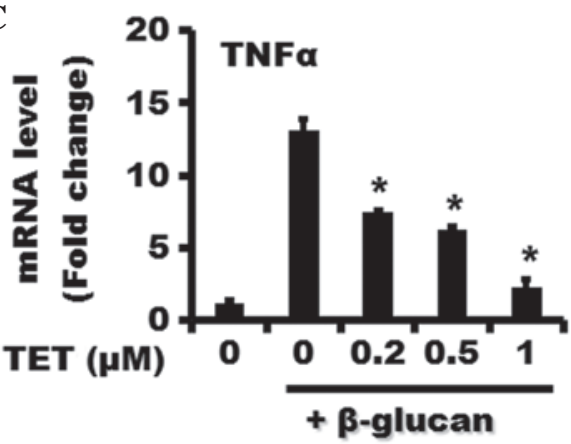

E

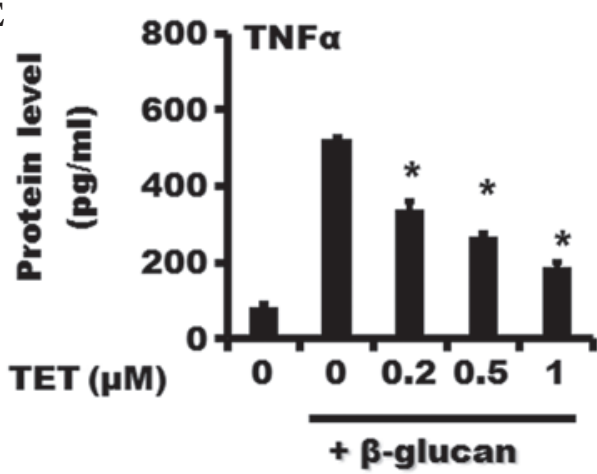

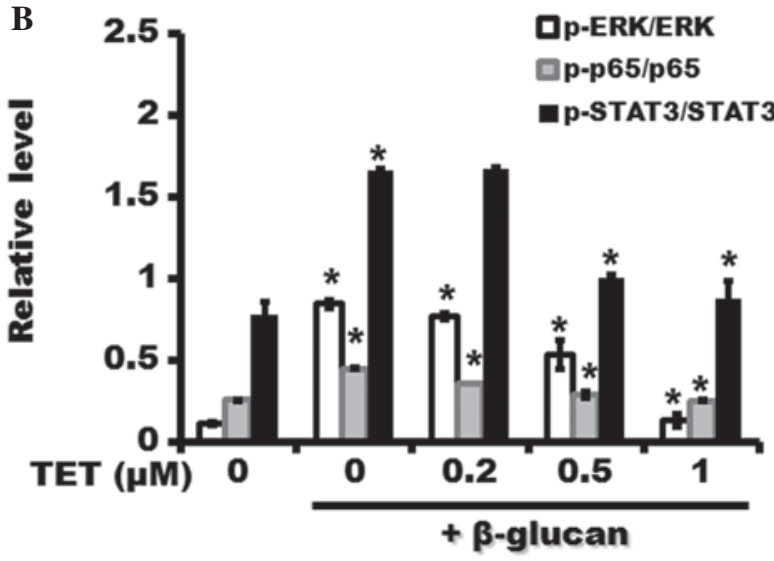

D

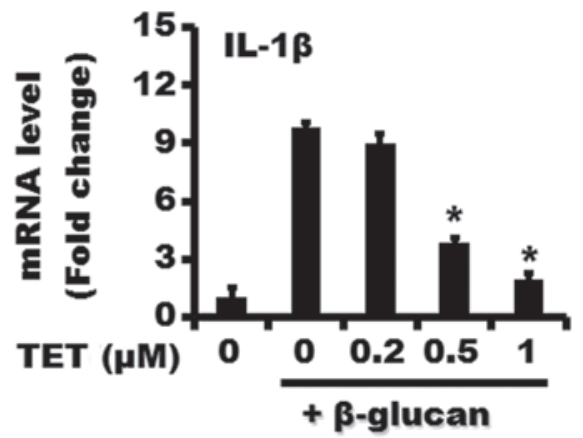

$\mathbf{F}$

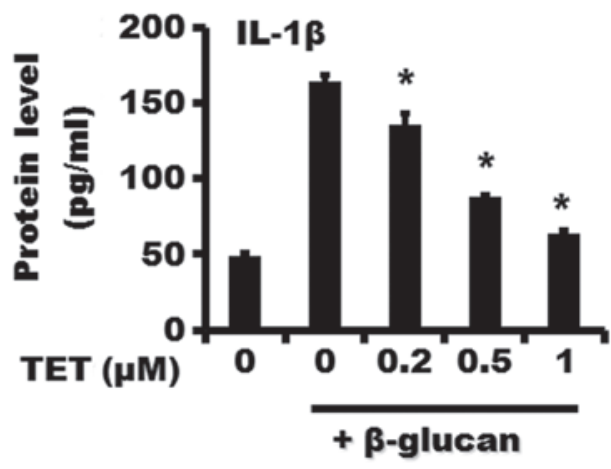

Figure 4. Effect of TET on $\beta$-glucan-induced activation of NF-кB, ERK and STAT3 pathways and upregulation of TNF $\alpha$ and IL-1 $\beta$ in THP-1 cells. THP-1 cells were pretreated with various concentrations of TET $(0,0.2,0.5$ and $1 \mu \mathrm{M})$ for $2 \mathrm{~h}$ followed by treatment with $\beta$-glucan $(100 \mu \mathrm{g} / \mathrm{ml})$ for $24 \mathrm{~h}$. (A) The expression of phosphorylated and total forms of p65, ERK and STAT3 proteins were examined by western blotting. (B) The relative expression of p-p65/p65, p-ERK/ERK and p-STAT3/STAT3 is shown. The expression of (C) TNF $\alpha$ and (D) IL-1 $\beta$ in THP-1 cells was examined by using reverse transcription-quantitative polymerase chain reaction. The expression of (E) TNF $\alpha$ and (F) IL-1 $\beta$ proteins in THP-1 cells was examined using an enzyme-linked immunosorbent assay. " $\mathrm{P}<0.05$, compared with control. TET, tetrandrine; ERK, extracellular signal-regulated kinase; NF- $\mathrm{kB}$, nuclear factor- $\mathrm{\kappa B}$; STAT3, signal transducer and activator of transcription 3; TNF $\alpha$, tumor necrosis factor- $\alpha$; IL, interleukin; p-, phosphorylated; GAPDH, glyceraldehyde 3-phosphate dehydrogenase; T-, total.

Statistical analysis. Data are presented as the mean \pm standard deviation. Statistical comparisons between two different treatments were analyzed using Student's t-test. Differences among more than two groups were tested by one-way analysis of variance, followed by a post-hoc test. $\mathrm{P}<0.05$ was considered to indicate a statistically significant difference.

\section{Results}

TET does not influence cell viability of macrophages at optimal concentrations. The molecular structure of TET is shown in Fig. 1A. To investigate the cytotoxic effect of TET on macrophages, Raw264.7 macrophages and THP-1 cells were treated with TET at various concentrations (ranging from 0 to $10 \mu \mathrm{M}$ ) for $24 \mathrm{~h}$. Cell viability was assessed using an MTT assay. As shown in Fig. 1B and C, TET at 0.2, 0.5, 1.0, 2.0, 5.0 and $10.0 \mu \mathrm{M}$ resulted in $99.15 \pm 3.74,109.70 \pm 2.66,98.40 \pm 2.36$, $41.86 \pm 2.32,13.39 \pm 0.38$ and $12.28 \pm 0.71 \%$ of RAW 264.7 macrophages surviving, respectively, and $99.39 \pm 1.28,92.50 \pm 1.07$, $85.17 \pm 1.01,68.35 \pm 2.20,37.88 \pm 0.52$ and $25.32 \pm 0.24 \%$ of THP-1 cells surviving, respectively. TET showed no cytotoxicity at a relatively low concentration (ranging from 0.2 to $1.0 \mu \mathrm{M})$. Consistent with the results of the MTT assay, TET at a relatively low concentration did not induce significant cell apoptosis (Fig. 2). Therefore, TET at a concentration ranging from 0.2 to $1 \mu \mathrm{M}$ was used in the following experiments.

$\beta$-glucan activates $N F-\kappa B, E R K$ and STAT3 signaling pathways and induces TNF $\alpha$ and IL-1 $\beta$ expression in THP-1 cells. The phosphorylation of p65, which was considered a marker 


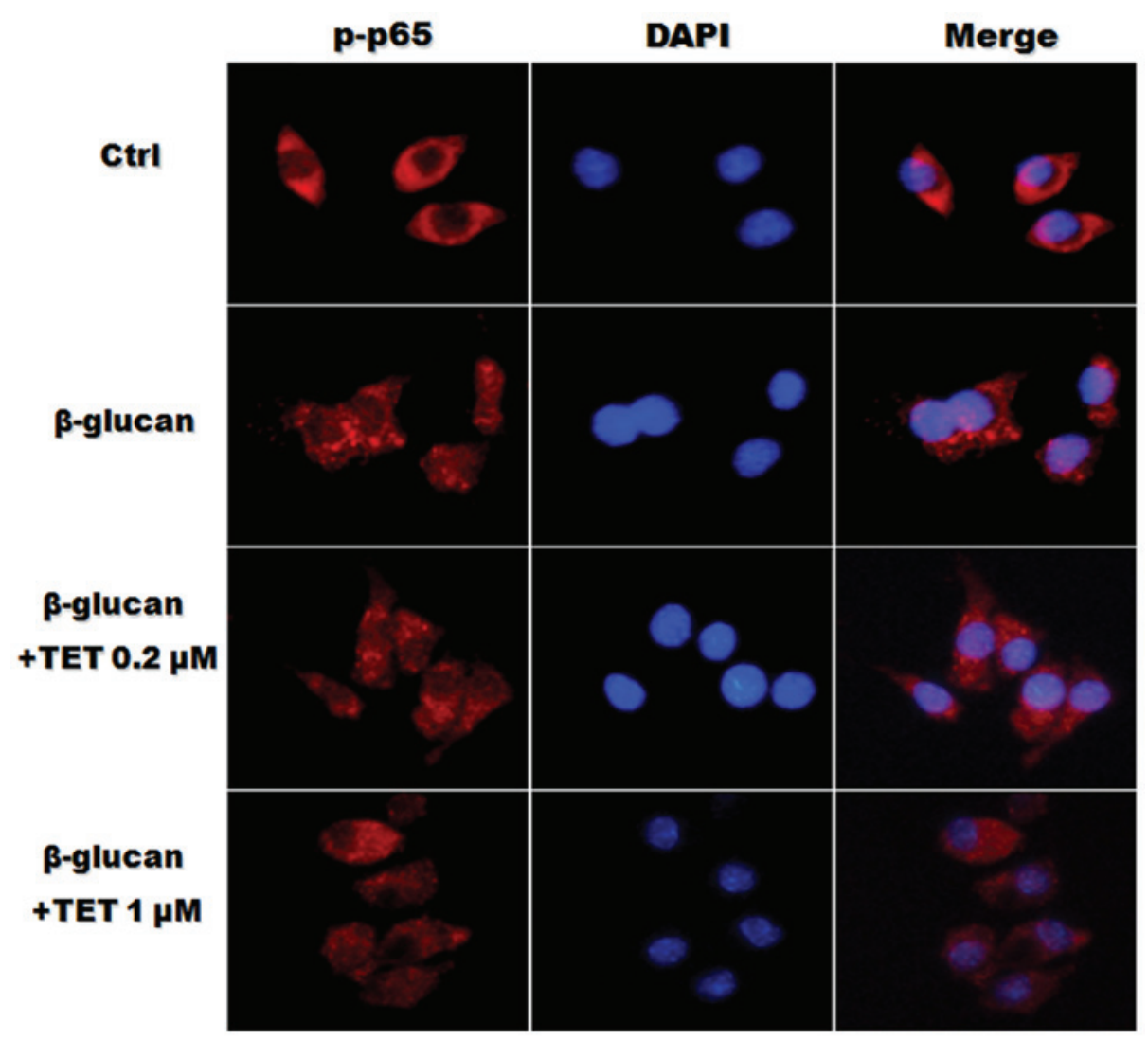

Figure 5. Effect of TET on $\beta$-glucan-induced nuclear translocation of NF-кB p65 in Raw264.7 macrophages. Raw264.7 macrophages seeded on coverlips were pretreated with various concentrations of TET $(0,0.2$ and $1 \mu \mathrm{M})$ for $2 \mathrm{~h}$ followed by treatment with $\beta$-glucan $(100 \mu \mathrm{g} / \mathrm{ml})$ for $24 \mathrm{~h}$. The localization of phosphorylated p65 was examined by using immunofluorescent staining. The cells were counterstained with DAPI to show nuclei (magnification, x100). p-, phosphorylated; TET, tetrandrine.

of NF- $\mathrm{kB}$ activation, was markedly increased after treatment with $\beta$-glucan compared with control (Fig. 3A and B). $\beta$-glucan also stimulated the phosphorylation of ERK and STAT3. To investigate the functional role of $\beta$-glucan on macrophages, the expression of TNF $\alpha$ and IL-1 $\beta$ mRNA was determined using RT-qPCR. As shown in Fig. $3 \mathrm{C}$ and D, the treatment with $\beta$-glucan dose-dependently induced the expression of TNF $\alpha$ and IL-1 $\beta$ mRNA in THP-1 cells. The expression of TNF $\alpha$ and IL- $1 \beta$ proteins was investigated using ELISA assays. $\beta$-glucan treatment was shown to upregulate the protein levels of TNFa and IL-1 $\beta$ in THP-1 cells (Fig. 3E and F).

TET suppresses $\beta$-glucan-induced activation of NF- $\kappa B, E R K$ and STAT3 signaling pathways and attenuates the expression of TNF $\alpha$ and IL-1 $\beta$ in $\beta$-glucan-stimulated THP-1 cells. The effect of TET on NF-кB, ERK and STAT3 activities in $\beta$-glucan-treated THP-1 cells was then investigated. It was demonstrated that TET pretreatment significantly inhibited the induced expression of p-p65, p-ERK and p-STAT3 by $\beta$-glucan treatment in a dose-dependent manner (Fig. 4A and B). $\beta$-glucan-induced upregulation of TNF $\alpha$ and IL-1 $\beta$ mRNA levels in THP-1 cells was inhibited by TET pretreatment (Fig. 4C and D). In addition, the increase in TNF $\alpha$ and IL-1 $\beta$ protein expression in THP- 1 cells by $\beta$-glucan was also reversed by TET pretreatment (Fig. 4E and F).

TET inhibits the nuclear translocation of $N F-\kappa B$ in $\beta$-glucan-stimulated RAW264.7 macrophages. The increased nuclear translocation of NF- $\mathrm{KB}$ is an indicator of activation. To further demonstrate that $\beta$-glucan stimulates the activation of NF- $\kappa B$, the distribution of NF- $\mathrm{BB}$ was detected in murine RAW264.7 macrophages using immunofluorescent staining. As shown in Fig. 5, NF- $\mathrm{kB}$ p65 protein was predominantly localized in the cytosol of RAW264.7 macrophages. $\beta$-glucan stimulation significantly increased the nuclear translocation of NF-kB protein. However, pretreatment with TET reduced the nuclear translocation of NF- $\kappa B$ in $\beta$-glucan-stimulated RAW264.7 macrophages.

TET suppresses the activation of $N F-\kappa B, E R K$ and STAT3 signaling pathways in $\beta$-glucan-stimulated RAW264.7 macrophages. To further confirm the inhibitory role of TET in macrophage activation, murine RAW264.7 macrophages with $\beta$-glucan were treated in the presence or absence of TET. As shown in Fig. 6, treatment with $\beta$-glucan also induced the activation of NF- $\mathrm{B}, \mathrm{ERK}$ and STAT3 signaling pathways in RAW264.7 macrophages. However, TET pretreatment significantly suppressed the activation of NF- $\mathrm{B}$, ERK and STAT3 signaling pathways by $\beta$-glucan.

\section{Discussion}

$\beta$-glucan is a heterogeneous group of glucose polymers found in the cell walls of fungi, bacteria and plants. $\beta$-glucan from fungi and bacteria activates immune cells, such as macrophages, to produce a wide spectrum of proinflammatory mediators, 
A

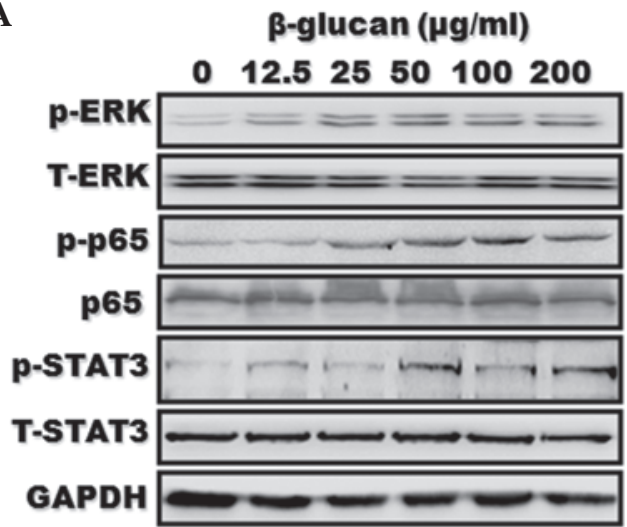

B

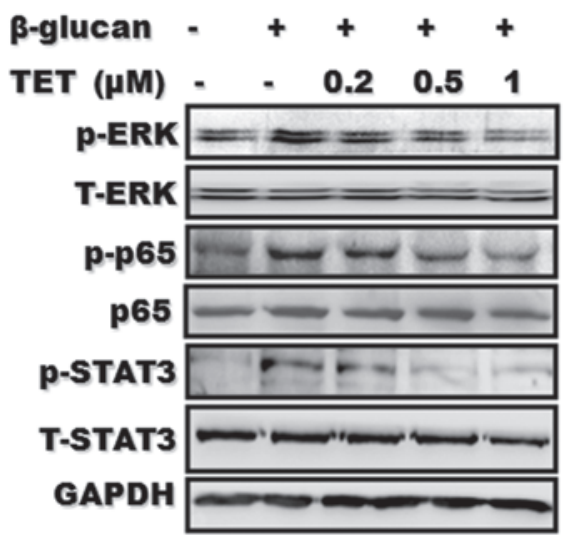

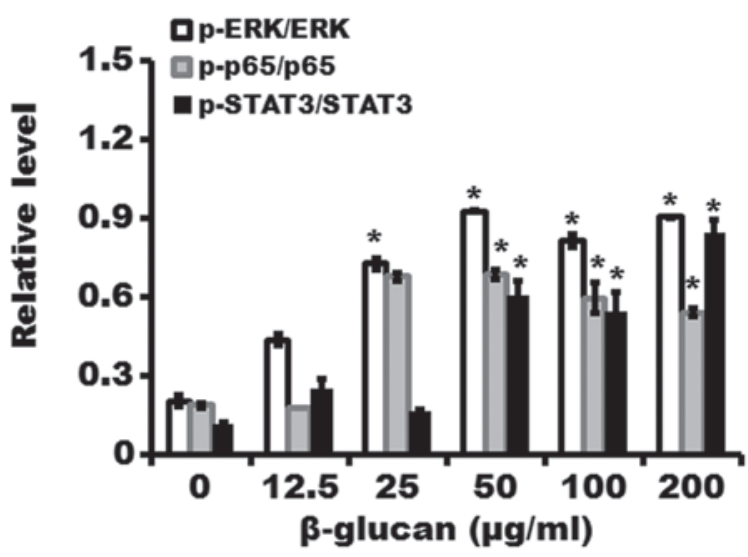

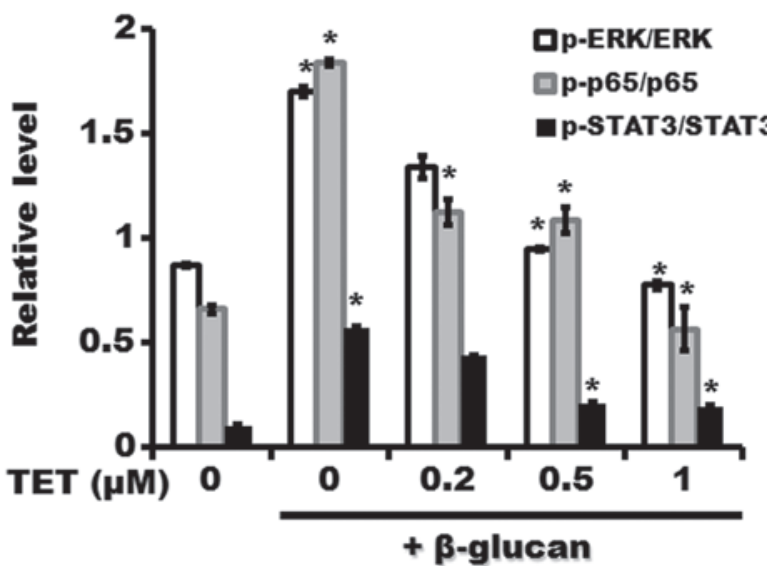

Figure 6. Effect of TET on $\beta$-glucan-induced activation of NF-kB, ERK and STAT3 pathways in Raw264.7 macrophages. (A) Raw264.7 macrophages were pretreated with various concentrations of $\beta$-glucan $(0,12.5,25,50,100$ and $200 \mu \mathrm{g} / \mathrm{ml})$ for $24 \mathrm{~h}$. The expression of phosphorylated and total forms of p65, ERK and STAT3 proteins were examined using western blotting. (B) Raw264.7 macrophages were pretreated with various concentrations of TET $(0,0.2,0.5$ and $1 \mu \mathrm{M})$ for $2 \mathrm{~h}$ followed by treatment with $\beta$-glucan $(100 \mu \mathrm{g} / \mathrm{ml})$ for $24 \mathrm{~h}$. The expression of phosphorylated and total forms of p65, ERK and STAT3 proteins was examined by using western blotting. "P<0.05, compared with control. TET, tetrandrine; ERK, extracellular signal-regulated kinase; STAT3, signal transducer and activator of transcription 3; p-, phosphorylated; GAPDH, glyceraldehyde 3-phosphate dehydrogenase.

which cause cell damage and tissue injury. $\beta$-glucan-induced production of proinflammatory cytokines has been considered to be critical in the pathogenesis of inflammatory diseases. In this study, it was demonstrated that TET, a compound isolated from a Chinese herb, attenuated $\beta$-glucan-induced inflammatory responses in murine and human macrophages without exhibiting cytotoxic effects. Given the broad biological activities of TET, the findings may provide a novel opportunity for $\beta$-glucan-associated inflammatory disease treatment.

Macrophage activation has been implicated in numerous inflammatory diseases. In vitro and in vivo studies have shown that $\beta$-glucan is able to stimulate the functional activation of macrophages. In this study, the effects of TET, a major pharmacologically-active compound of Chinese herb Stephania tetrandra S Moore on macrophage activation induced by $\beta$-glucan was investigated. Murine- and human-derived macrophages pretreated with TET were stimulated by $\beta$-glucan in vitro. Data showed that TET inhibited the expression of inflammatory mediators including TNF- $\alpha$ and IL-1 $\beta$ in $\beta$-glucan-stimulated macrophages. The suppressive roles of TET may be attributed to its inhibitory effect on the NF- $\kappa \mathrm{B}$ pathway during macrophage activation. TET has been previously suggested to exhibit immunosuppressive properties

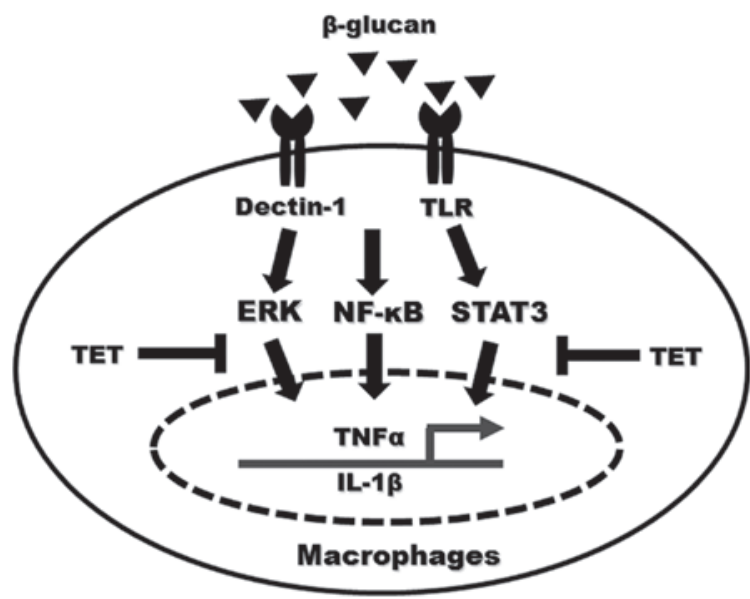

Figure 7. Proposed model for the inhibitory role of TET on $\beta$-glucan-induced macrophage activation. TET, tetrandrine; TLR, toll-like receptor; ERK,

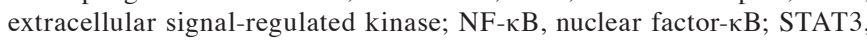
signal transducer and activator of transcription 3; TNF $\alpha$, tumor necrosis factor- $\alpha$; IL, interleukin.

in vitro and in vivo. To the best of our knowledge, the present study demonstrated for the first time that TET showed significant suppressive effects on $\beta$-glucan-induced macrophage 
activation. Furthermore, this study has also shown that the inhibitory effect of TET is not due to its direct cytotoxicity since the doses of used in this study did not affect the viability of macrophages.

TNF $\alpha$ and IL-1 $\beta$ are produced at the early stages of inflammation. The levels of TNF- $\alpha$ and IL-1 $\beta$ are elevated in the majority of inflammatory diseases and are involved in the pathology of inflammation. The elevated production of TNF- $\alpha$ and IL-1 $\beta$ leads to local inflammation and tissue damage. In this study, it was demonstrated that $\beta$-glucan induced the expression of TNF- $\alpha$ and IL- $1 \beta$ in a concentration-dependent manner in macrophages. TET pretreatment could suppress the induction of TNF- $\alpha$ and IL-1 $\beta$, suggesting that TET is a potent inhibitor for $\beta$-glucan-induced expression of inflammatory mediators in macrophages.

$\mathrm{NF}-\kappa \mathrm{B}$ is an important transcriptional factor for regulating the expression of inflammatory mediators. NF- $\kappa \mathrm{B}$ is key in the release of proinflammatory cytokines. $\mathrm{NF}-\kappa \mathrm{B}$ is retained in the cytosol by inhibitory proteins in resting cells. Once stimulated, NF- $\kappa \mathrm{B}$ translocates from the cytosol to the nucleus where it binds to the regulatory element in the promoter region of its target genes (such as TNF $\alpha$ and IL-1 $)$ ). In the present study, it was demonstrated that $\beta$-glucan treatment significantly increased the expression of phosphorylated p65, which is a subunit of $\mathrm{NF}-\kappa \mathrm{B}$ transcription factor and is considered to be a marker of $N F-\kappa B$ activation, and significantly upregulated the expression of TNF $\alpha$ and IL-1 $\beta$. In previous studies, TET has been found to inhibit NF- $\kappa \mathrm{B}$ activation and nuclear translocation in several cell and animal models $(6,13,14)$. Studies have demonstrated that the inhibitory effect of TET on NF- $\kappa$ B activation can be attributed to its ability to prevent the degradation of $\mathrm{I} \kappa \mathrm{B} \alpha$ (a cytoplasmic inhibitor of NF- $\kappa \mathrm{B}$ ) and inhibit nuclear translocation of p65 (15). In this study, it was demonstrated that TET exerted its inhibitory effect on $\beta$-glucan-induced macrophage activation by inhibiting the nuclear translocation of p65, leading to the inactivation of its downstream target genes. In addition, the ERK and STAT3 signaling pathways have been shown to be critical in regulating the release of proinflammatory cytokines. In this study it was demonstrated that the phosphorylation of ERK and STAT3 was markedly inhibited by TET. ERK and STAT3 signaling pathways have been suggested to control the activation of NF- $\kappa \mathrm{B}$ in activated cells. Therefore, these results indicate that $\beta$-glucan-induced activation of NF- $\mathrm{NB}, \mathrm{ERK}$ and STAT3 cooperatively regulate inflammatory cytokine expression in macrophages, and that TET exerts inhibitory roles by suppressing NF- $\mathrm{B}$, ERK and STAT3 activation (Fig. 7).

In conclusion, the results suggest that TET suppresses $\beta$-glucan-induced macrophage activation and reduces the release of inflammatory mediators. TET exerts the suppressive effects through the inhibition of NF- $\kappa \mathrm{B}$, ERK and STAT3 pathways. These findings, together with those of the previous studies, suggest the potential use of TET in the treatment of $\beta$-glucan-associated inflammatory diseases.

\section{Acknowledgements}

This study was supported by the Technology Support Program of Zhenjiang (grant no. SH2013062).

\section{References}

1. Qin R, Shen H, Cao Y, Fang Y, Li H, Chen Q and Xu W: Tetrandrine induces mitochondria-mediated apoptosis in human gastric cancer BGC-823 cells. PLoS One 8: e76486, 2013.

2. Qiu W, Su M, Xie F, Ai J, Ren Y, Zhang J, Guan R, He W, Gong Y and Guo Y: Tetrandrine blocks autophagic flux and induces apoptosis via energetic impairment in cancer cells. Cell Death Dis 5: e1123, 2014.

3. He FQ, Qiu BY, Li TK, Xie Q, Cui de J, Huang XL and Gan HT: Tetrandrine suppresses amyloid- $\beta$-induced inflammatory cytokines by inhibiting NF- $\kappa \mathrm{B}$ pathway in murine BV2 microglial cells. Int Immunopharmacol 11: 1220-1225, 2011.

4. Xue Y, Wang Y, Feng DC, Xiao BG and Xu LY: Tetrandrine suppresses lipopolysaccharide-induced microglial activation by inhibiting NF-kappaB pathway. Acta Pharmacol Sin 29: 245-251, 2008.

5. Dang Y, Xu Y, Wu W, Li W, Sun Y, Yang J, Zhu Y and Zhang C: Tetrandrine suppresses lipopolysaccharide-induced microglial activation by inhibiting NF- $\mathrm{kB}$ and ERK signaling pathways in BV2 cells. PLoS One 9: e102522, 2014.

6. Kang OH, An HJ, Kim SB, Mun SH, Seo YS, Joung DK, Choi JG, Shin DW and Kwon DY: Tetrandrine suppresses pro-inflammatory mediators in PMA plus A23187-induced HMC-1 cells. Int J Mol Med 33: 1335-1340, 2014.

7. Chan GC, Chan WK and Sze DM: The effects of beta-glucan on human immune and cancer cells. J Hematol Oncol 2: 25, 2009.

8. Li X, Wang J, Wang W, Liu C, Sun S, Gu J, Wang X, Boraschi D, Huang Y and Qu D: Immunomodulatory activity of a novel, synthetic beta-glucan ( $\beta$-glu6) in murine macrophages and human peripheral blood mononuclear cells. PLoS One 8: e80399, 2013.

9. Karumuthil-Melethil S, Gudi R, Johnson BM, Perez N and Vasu C: Fungal $\beta$-glucan, a dectin-1 ligand, promotes protection from type 1 diabetes by inducing regulatory innate immune response. J Immunol 193: 3308-3321, 2014.

10. Brown GD, Taylor PR, Reid DM, Willment JA, Williams DL, Martinez-Pomares L, Wong SY and Gordon S: Dectin-1 is a major beta-glucan receptor on macrophages. J Exp Med 196: 407-412, 2002.

11. Yadav M and Schorey JS: The beta-glucan receptor dectin-1 functions together with TLR2 to mediate macrophage activation by mycobacteria. Blood 108: 3168-3175, 2006.

12. Chang ZQ, Lee JS, Gebru E, Hong JH, Jung HK, Jo WS and Park SC: Mechanism of macrophage activation induced by beta-glucan produced from Paenibacillus polymyxa JB115. Biochem Biophys Res Commun 391: 1358-1362, 2010.

13. Zhao H, Luo F, Li H, Zhang L, Yi Y and Wan J: Antinociceptive effect of tetrandrine on LPS-induced hyperalgesia via the inhibition of IKK $\beta$ phosphorylation and the COX-2/PGE 2 pathway in mice. PLoS One 9: e94586, 2014.

14. Wang QS, Cui YL, Gao LN, Guo Y, Li RX and Zhang XZ: Reduction of the pro-inflammatory response by tetrandrine-loading poly (L-lactic acid) films in vitro and in vivo. J Biomed Mater Res A 102: 4098-4107, 2014.

15. Lin ST, Wang Y, Xue Y, Feng DC, Xu Y and Xu LY: Tetrandrine suppresses LPS-induced astrocyte activation via modulating IKKs-IkappaBalpha-NF-kappaB signaling pathway. Mol Cell Biochem 315: 41-49, 2008. 